\title{
Experiences in the development of electronic care plans for the management of comorbidities
}

\author{
E. Lozano ${ }^{\star 1}$, M. Marcos ${ }^{2}$, B. Martínez-Salvador ${ }^{2}$, A. Alonso ${ }^{1}$, and J.R. Alonso ${ }^{1}$ \\ 1 Hospital Clínic, Barcelona, Spain, \\ 2 Universitat Jaume I, Castellón, Spain
}

\begin{abstract}
Recent studies have shown that care plans with comprehensive home interventions can be effective in the management of chronic patients. Evidence also exists about the importance of tailoring these care plans to patients, by integrating comorbidities. In this context, the development, implementation, outcome analysis, and reengineering of care plans adapted to particular patient groups earn relevance. We are concerned with the development and reengineering of electronic care plans dealing with comorbidities. Our hypothesis is that a library of reusable care plan components can facilitate these tasks. To confirm this hypothesis we have carried out an experiment consisting in developing a library of care plan components for the management of patients with $\mathrm{COPD}^{3}$ or $\mathrm{CHF}^{4}$, and next building a care plan for stable COPD\&CHF patients by (re)using these components. In this paper we report on this experiment.
\end{abstract}

Key words: Care plans, knowledge modelling, reusable components, guideline representation languages, PROforma.

\section{Introduction}

Recent studies have shown that care plans ${ }^{5}$ with comprehensive home interventions can be effective in the management of chronic patients [2]. These plans include interventions that do not require a direct supervision by the physician, such as answering of questionnaires by the patient, or home visits and/or phone consultations by nurses (with access to the physician at the hospital for remote supervision). Most of these models, though, are exclusively targeted on one disease (i.e. COPD, CHF, Diabetes) in isolation. Evidence exists about the importance of integrating comorbidities in such models as a way of making them better tailored to patients. In such home-based health-care scenarios, the development and outcome analysis of care plans tailored to the management of particular

\footnotetext{
* This work has been supported by the Spanish Ministry of Science and Technology, through the research project TIN2006-15453 - HYGIA.

${ }^{3}$ Chronic obstructive pulmonary disease.

${ }^{4}$ Chronic heart failure.

${ }^{5}$ We use the term care plan to refer to a high level description of the overall plan of actions for the care of a patient, constructed from the basis of one or more guidelines that the patient should follow, similarly to the definition by Barretto [1].
} 
patient groups earn relevance. Thus, if a concrete care plan does not prove to be effective according to predefined indicators it will have to be reengineered and implemented in its new form.

Several Artificial Intelligence (AI) tools can be used to facilitate the different phases of the process sketched above, generally speaking, care plan development, implementation, outcome analysis, and reengineering. The objectives of the HYGIA project [3] are in line with this overall goal. In HYGIA we tackle the development of care plans in electronic format using existing AI languages for the representation of medical guidelines. Apart from serving as a basis for further development of dedicated decision support systems, the representation of care plans in these languages is important in terms of health-care knowledge capitalization.

Within the HYGIA project, we approach the development of care plans as a knowledge acquisition task during which the necessary knowledge is collected from different sources, mainly selected medical guidelines and experts' knowledge. At the same time, we concentrate on care plans for the treatment of comorbidities. In this framework, our hypothesis is that a library of reusable care plan components can facilitate the development and reengineering of electronic care plans, even directly by medical experts [4].

To validate the above hypothesis, we have worked on a library of care plan components incorporating experts' knowledge about the necessary home interventions to manage patients under particular conditions, namely COPD or CHF. Then we have used these components to build a care plan for a specific group of patients, namely patients with both COPD and CHF who are stable, and with a clinical goal in mind, which is the prevention of exacerbations. In this paper we report on this experiment.

It is important to note that at this stage we do not aim at providing a fully functional tool to support care plan developers. Rather, this work should be considered as a "proof of concept" to validate our hypothesis, using existing guideline representation languages and tools. Consequently, the development of more specific tools with advanced features (e.g. web-based, collaborative) is beyond the scope of this work.

The structure of the paper is as follows. First section 2 details the experimental setting, including the modelling strategy that has been adopted and the guideline representation language (and tools) used. Then section 3 gives details on the care plan components that have been developed. Afterwards section 4 describes the development of the care plan for the management of COPD\&CHF patients, including difficult issues and results. Finally section 5 presents our conclusions and points to the future work.

\section{Experimental framework}

Our experiment consists in the development of a care plan in electronic format for the prevention of exacerbations in stable COPD\&CHF patients, care plan which is partly based on home-based interventions. The need for such a spe- 
cialised care plan, including the possibility of care plan reengineering, originates from ongoing clinical research at the Hospital Clínic of Barcelona [5], [2]. Likewise, the domiciliary interventions to be included in the care plan have been established by and are specific to this hospital. Finally, the recommendations for the diagnosis and treatment of COPD and CHF conditions are based on the recommendations from the guideline of the Global Initiative for Chronic Obstructive Lung Disease (GOLD) [6] and the guideline for the diagnosis and treatment of CHF of the European Society of Cardiology (ESC) [7], respectively.

With regard to the care plan itself, note that we entirely rely on the expertise of researchers of Hospital Clínic. Thus, all the medically relevant aspects (e.g. severity of the disease) have been specified in the care plan by the hospital experts. Besides, issues like the clinical validation of the care plan using cohort data is considered to be outside the scope of this work. Instead we focus on the development aspects from a knowledge engineering perspective, with the goal of facilitating care plan reengineering (as determined by the developers).

In this light we have identified two important issues related to the experimental framework. First, it must be clarified to which extent the care plan knowledge is based on the experts' knowledge and the guidelines, to determine the acquisition and subsequent modelling strategies. Second, it is important to decide on the appropriate guideline representation language and tools, practicable for the purposes of care plan development and reengineering from components. The following subsections deal with these issues.

\subsection{Knowledge acquisition framework}

As mentioned before, the knowledge necessary for the development of the care plan has both the hospital experts and the GOLD and ESC guidelines as sources. With the purpose of determining the scope of each of these knowledge sources, initial interviews were conducted. As result, it became clear that the care plan is structured into three main phases, namely admission, re-evaluation and followup, each of them including interventions for both COPD and CHF.

The admission phase deals with the initial assessment of the disease and determination of the treatment, as well as with the complementary interventions performed in the Hospital Clínic (mainly completion of questionnaires) to determine if the patient can be enrolled in the care plan. The knowledge for the interventions in this phase comes mainly from the guidelines, in the case of the assessment and the determination of the treatment, but also from the hospital experts, in the case of the complementary actions. The re-evaluation is a short phase carried out at the patient's home. In this phase, the data provided by the patient in the questionnaires (e.g. about his/her knowledge of the disease) are revised, and educational directions are given based on the weak points detected. Finally, in the follow-up phase the status of the patient is periodically assessed through anamnesis and clinical examination. In case a critical condition is detected, such as cardiac decompensation, the patient should leave the care plan and enroll in a more appropriate one. Another important intervention in this phase is the revision of the treatment, if needed. The knowledge for the 
re-evaluation and follow-up phases and comes mainly from the experts, specially in the case of interventions for the management of comorbidities.

Therefore, we are in a context in which guideline knowledge must be combined with experts' knowledge in a care plan structured according to the three phases described above, which bears little relation to the GOLD and ESC guidelines. In such a context, we have opted for modelling components to be used as care plan building blocks, instead of modelling the guidelines and then selecting and adapting parts thereof. The former approach can be named as "care plan oriented", as opposed to the latter "guideline oriented" one. Figure 1 shows a schematic view of the "care plan oriented" approach we have followed.

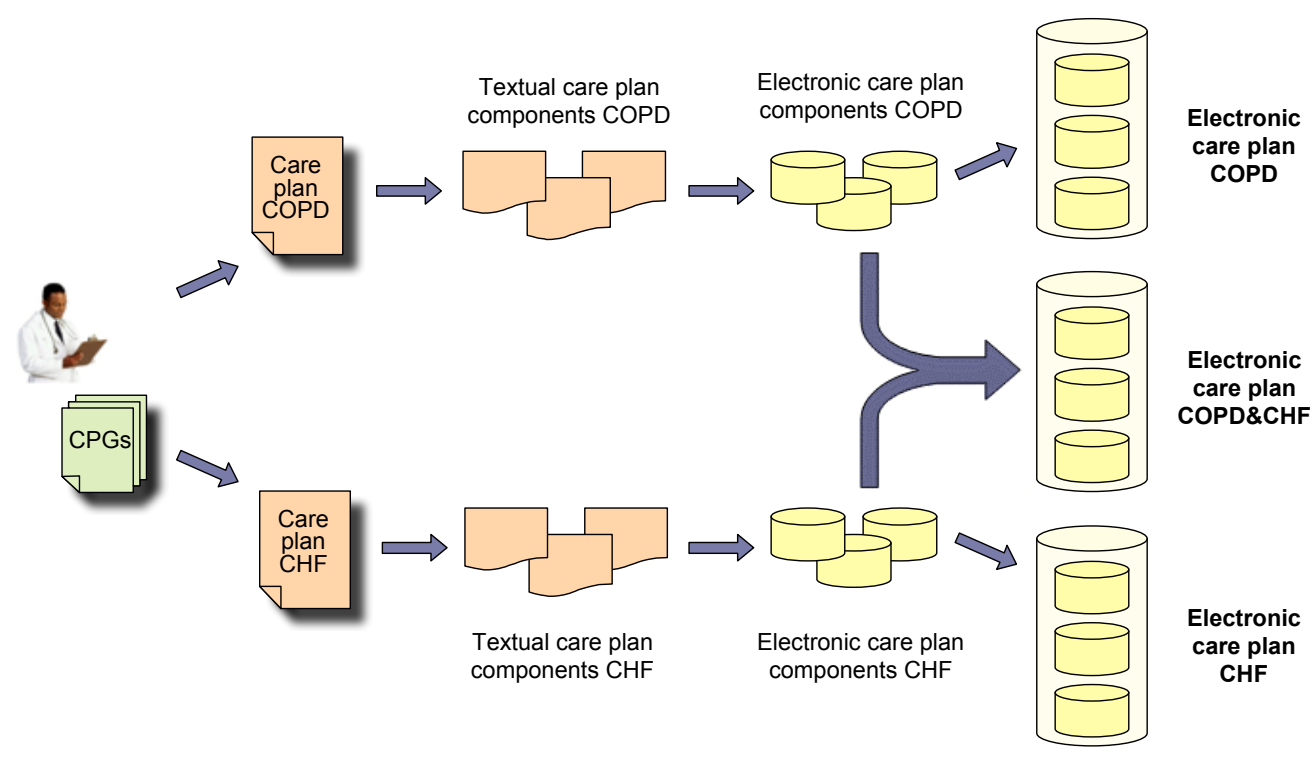

Fig. 1. Strategy for the development of a care plan for COPD\&CHF management.

With a "care plan oriented" approach the acquisition\&modelling strategy follows a path like:

textual care plan (with expert and guideline knowledge) $\rightarrow$ textual care plan components $\rightarrow$ electronic care plan components $\rightarrow$ electronic care plan

instead of:

textual guidelines $\rightarrow$ electronic guidelines $\rightarrow$ electronic care plan (with expert knowledge)

The distinctive features of the "care plan oriented" approach are (1) the central role of the knowledge needed for the care plan, (2) the focus on care plan 
building blocks (or components), and (3) the point of the process at which the specific expert knowledge is fed, which is earlier than in the case of the "guideline oriented" approach.

Lastly, and with the purpose of facilitating the development task (while increasing the possibilities of reuse of the care plan components in the library), we have opted for first modelling the care plan components for the management of COPD and CHF separately (both for stable patients), then building the respective care plans, and finally developing the care plan for the management of COPD\&CHF patients. Figure 1 also illustrates the creation of separate COPD and CHF care plans prior to the development of the overall one.

\subsection{Guideline representation language}

In addition to the acquisition\&modelling strategy, the choice of the knowledge representation language is another important aspect of our experiment. Within the HYGIA project we have committed ourselves to the use of one of the existing guideline representation languages. Among the leading languages for guideline representation cited in recent reviews [8], [9], [10], we examined Asbru, GLIF, PROforma, and SAGE. The selection was based on a set of requirements, mainly the availability of a modelling tool with graphical elements, the availability of a suitable execution engine, and the adequacy of the modelling\&execution tools for the development of both care plan components and care plans of some size (this includes e.g. the possibility of nesting of tasks). The PROforma language was chosen based on these requirements, and also taking into consideration the expertise of the participants in the HYGIA project.

The PROforma language was developed at the Imperial Cancer Research Fund by Fox and colleagues [9], [11]. In PROforma a guideline is modelled in terms of tasks hierarchically organised in plans. PROforma tasks fall into four categories, namely: actions, enquiries, decisions and plans. Plans are used to group together other tasks e.g. so that they are performed at the same time. PROforma processes can be represented as directed graphs in which different types of nodes represent tasks and arcs represent scheduling constraints. Tasks may have a number of properties that determine the way they must be executed. For instance, plans can have preconditions and task scheduling constraints (these properties are shared with the rest of task categories), but also termination and abort conditions.

With respect to the tools, we have used the Tallis toolset [12]. Tallis includes two applications: the composer, which is a graphical environment for the creation and editing of guidelines, and the tester, which is an integrated tool for enacting guidelines step by step. Sections 3 and 4 below describe the components and the care plans that have been developed as part of our experiment. These sections also include some examples of PROforma processes. 


\section{Care plan components for the management of COPD and $\mathrm{CHF}$}

As explained previously, we have first developed the care plan components for the management of COPD and CHF. This section includes examples taken from the COPD care plan. Figure 2 contains a screenshot of the Tallis tool showing the task network representing the COPD_management care plan. This network includes three plan nodes (represented as rounded boxes), one for each of the main phases described in section 2, together with the scheduling constraints among them (represented as directed arcs). In this case, the constraints impose a sequential control flow among the plans. Moreover, in figure 2 we can see a hierarchical decomposition of the PROforma tasks used in the description of the COPD care plan, among other things.

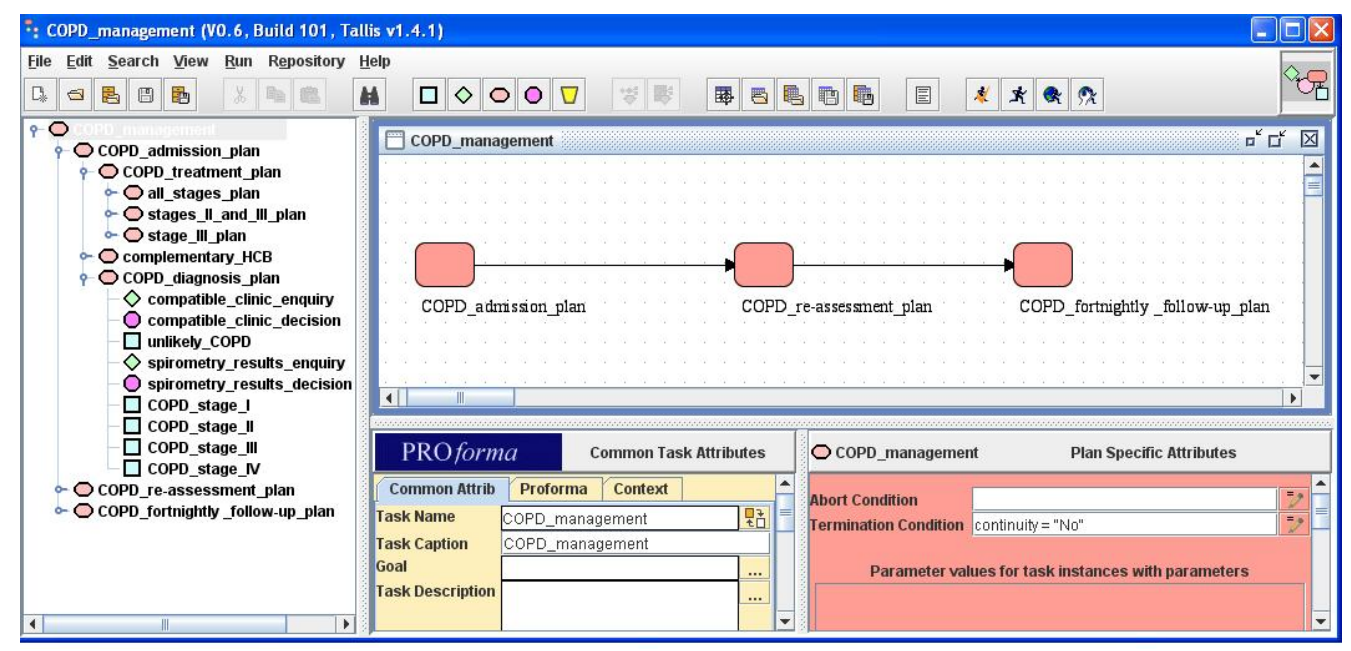

Fig. 2. PROforma model of a care plan for COPD management.

Figure 3 shows the task network pertaining to the COPD_diagnosis plan, which is part of the COPD_admission one. In this case the network includes enquiry nodes (represented as diamonds) to gather the necessary clinical data, and decision nodes (represented as circles) to subsequently confirm the COPD diagnosis and determine the severity of the disease. The latter has been modelled by means of action nodes (represented as boxes).

We posit that the PROforma plans in figure 2, as well as other plans at lower levels such as COPD_diagnosis (the details of which are shown in figure 3), constitute the components from which more complex care plans related to the COPD condition can be constructed. 


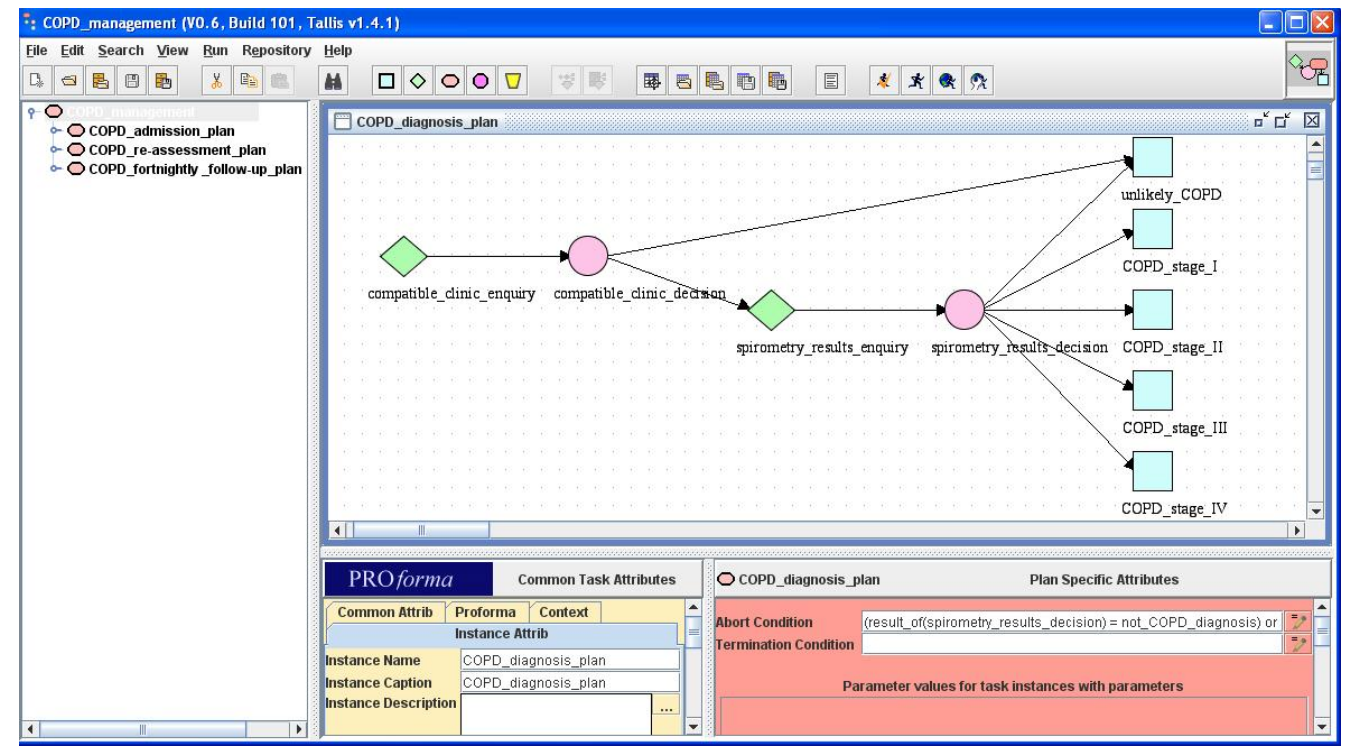

Fig. 3. PROforma model of COPD diagnosis.

\section{Care plan for the management of stable COPD\&CHF patients}

Based on the care plan components developed in the first place, we have constructed a care plan for the management of COPD\&CHF patients. At a high level, the care plan also consists of three main plans, namely admission, reevaluation and follow-up. Each of these plans has been developed by reusing different parts of the COPD and CHF plans, mostly whole plans but also different plan elements. The example below tries to illustrate this.

The PROforma network in figure 4 represents the plan COPD-CHF_management. This plan not only has the same structure as the plan in figure 2, but also contains the same definition for certain tasks (e.g. COPD_diagnosis and COPD_treatment, see the hierarchical decomposition in figure 4).

We have identified several scenarios regarding the reuse of care plan components. In some cases, e.g. in the case of the diagnosis and treatment plans, the PROforma plans have been directly reused (i.e. cut\&pasted) as off-the-shelf components. This implies that both the layout of the task network and the tasks themselves are reused. Otherwise, when direct reuse was not possible or not deemed appropriate for some reason, different solutions have been adopted. The reuse scenarios that we have identified are the following:

1. direct reuse of plan (task layout plus tasks) as an off-the-shelf component. 


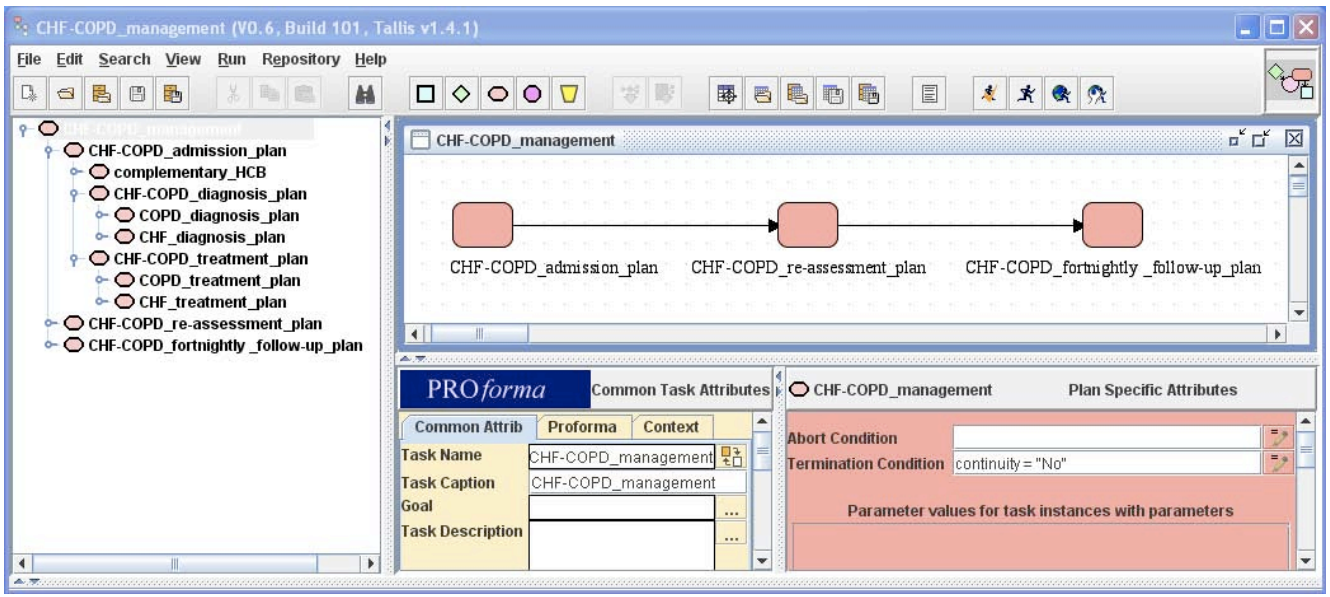

Fig. 4. PROforma model of a care plan for COPD\&CHF management.

2. reuse of plan (task layout plus tasks) with small changes, either (a) minor changes in tasks, e.g. changes in task preconditions, or (b) addition of a small number of tasks. In this scenario the original layout and tasks are preserved.

3. reuse of task layout with replacement of tasks by similar ones, mostly different versions thereof. Note that this scenario also covers the cases in which the resulting plan includes additional elements, provided that the original task layout is preserved.

In addition to direct reuse, note that the reuse of plans with small changes and the reuse of task layout with replacement of tasks (i.e. scenarios 2 and 3 ) in general do not require great effort and produce actual modelling benefits. The gain of reuse depends on the complexity and size of the object reused. To give an idea of the latter, the plans reused in our experiment contained 3 to 40 tasks.

As example of situation where the modelling effort was higher we can mention the adjustment made to the plan class_I_plan (therapy plan within CHF_treatment_plan for class I patients) so that it could be used in the COPD\&CHF care plan. Concretely, the part for the (CHF) beta-blocker therapy had to be adapted to cope with contraindications that apply to a subset of COPD patients. Although this example falls in scenario 2(a), it required further acquisition of specialised knowledge on comorbidities and hence it was considered harder. This has led us to the conclusion that plans should be documented with contraindications of the procedures they contain, to facilitate the reuse/adaptation in these situations.

Table 4 shows some numbers from our modelling experiment. The table lists the number of occurrences of the different reuse scenarios, together with the total number of (sub)tasks involved in the reuse (note that reusing a whole plan implies reusing all its subtasks) and the percentages with respect to the total number of tasks in the care plan. In this total we have disregarded duplicate plans (which 
would have been copied\&pasted anyway, with or without component reuse), resulting in 136 subtasks. Likewise, we are not looking at duplicate parts to count reuse occurrences. Finally, note that in some cases the number of involved subtaks is approximate, due to the nature of the reuse scenario.

Table 1. Reuse of tasks in the development of a care plan for COPD\&CHF management from components.

\begin{tabular}{|l|l|l|}
\hline Reuse scenario & $\begin{array}{l}\text { Number of occur- } \\
\text { rences }\end{array}$ & $\begin{array}{l}\text { Number of (sub)tasks } \\
\text { reused (and \% w.r.t. } \\
\text { total }^{a} \text { ) }\end{array}$ \\
\hline scenario 1, reuse of plan & 3 & $64(47 \%)$ \\
\hline $\begin{array}{l}\text { scenario 2, reuse of } \\
\text { plan w/small changes } \quad \text { (b) }\end{array}$ & 1 & $\begin{array}{l}41(30.1 \%) \\
19(14 \%)\end{array}$ \\
\hline $\begin{array}{l}\text { scenario 3, reuse of } \\
\text { task layout }\end{array}$ & 3 & $\mathrm{~N} / \mathrm{A}^{b}$ \\
\hline
\end{tabular}

${ }^{a}$ The total number of tasks (excluding duplicate plans) is 136 .

${ }^{b}$ Reuse of task layout relies on new subtasks.

In terms of the number of subtasks reused, the most important reuse scenarios are 1 and 2(a), i.e. direct reuse and reuse of plans with minor changes in tasks. All in all, mentioning that although other types of reuse are not so important in terms of subtasks reused, they occur in practice and prove to be important in care plan development (see e.g. the reuse of layout with task replacement in figure 4). Finally, pointing out that nearly all the plans are more or less based on previous components (over $90 \%$ of subtasks). Therefore, we can consider that the library of care plan components has simplified the construction of the COPD\&CHF care plan to a great extent.

\section{Conclusions}

Recent studies have shown that care plans with comprehensive home interventions can be effective in the management of chronic patients. Evidence also exists about the importance of tailoring these care plans to patients, by integrating comorbidities. In this context, the development, implementation, outcome analysis, and reengineering of care plans adapted to particular patient groups earn relevance.

We are concerned with the development and reengineering of electronic care plans dealing with comorbidities. Our hypothesis is that a library of reusable care plan components can facilitate these tasks. To confirm this hypothesis we have carried out an experiment consisting in developing a library of care plan components for the management of patients with COPD or CHF, and next building a care plan for stable COPD\&CHF patients by (re)using these components. For this purpose we have used the PROforma guideline representation language and its associated tools. 
We have succeeded in building a complete care plan for a practical application from the care plan components previously developed, as shown in section 4. This has been done by either directly reusing the components as they were, or by reusing parts of the description of these elements, e.g. the task layout. We consider that the reuse of care plan components has simplified the construction of the care plan to a great extent. On the downside we should mention that there are situations which require additional acquisition\&modelling work, even with component reuse. Still, this additional work can be preserved in the library, in the form of a new, more specialised care plan component. Section 4 includes an example of such situations, which is related to the fact that COPD and CHF are comorbidities.

The "care plan oriented" approach, focused on care plan modelling rather than on guideline modelling, has proven to be very useful at least in two respects. First, we have limited ourselves to modelling those guideline parts which were required for the care plan, disregarding the rest. E.g. the parts dedicated to the management of very severe COPD patients have not been modelled, because they are outside the scope of the care plan. And second, the components are designed for use in care plans for home-based management of patients and thus incorporate knowledge not covered in the guidelines. As result, the components incorporate specific knowledge that otherwise would have to be added in later stages.

With respect to the language and tools, we can say that they are well adapted to our purposes. The PROforma language has served to describe the care plan knowledge in a convenient manner. On the negative side we can mention the lack of language elements to describe concepts which are needed in care plans, such as roles and actors. As for the tools, we can highlight as advantages the graphical editing as well as the integration with an execution engine (the tester), which facilitates care plan debugging. An aspect where improvements could be made is the support for cut\&paste of tasks, which is crucial for our purposes. A better support is to be expected in the commercial version of PROforma tools.

As future work we plan to carry out the same exercise with different combinations of diseases, building care plans to deal with COPD, CHF, and/or Diabetes. With a wider case study we will be better placed to determine e.g. how the library components must be documented to facilitate reuse/adaptation (e.g. with contraindications of the procedures within a plan). In parallel, we will explore other solutions to the representation of electronic care plans, possibly different from guideline languages.

In addition to the above, we will study the suitability of the framework for the development and reengineering of electronic care plans directly by care plan developers. Since the languages and tools we use come from the AI in Medicine field, it is crucial to ensure that they can be used by medical experts with no effort. Related to this issue and to the usability of electronic care plans developed, we plan to put into operation a decision support system to monitor a set of indicators as defined by care plan developers. In case these indicators show that the care plan is not appropriate for the target patient group, it will 
have to be reengineered and integrated into the decision support system in its new form.

\section{References}

1. Barretto, S.A.: Designing Guideline-based Workflow-integrated Electronic Health Records. PhD thesis, School of Computer and Information Science, University of South Australia (2005)

2. Casas, A., Troosters, T., Garcia-Aymerich, J., Roca, J., Hernández, C., Alonso, A., del Pozo, F., de Toledo, P., Antó, J., Rodríguez-Roisín, R., Decramer, M.: Integrated care prevents hospitalisations for exacerbations in COPD patients. European Respiratory Journal 28 (2006) 123-130

3. HYGIA Project. http://banzai-deim.urv.net/ riano/TIN2006-15453/. Date of access: May 2009

4. Alonso, A., Marcos, M., Alonso, J., Gelabert, G., Martínez-Salvador, B., Riaño, D., Taboada, M.: A knowledge-acquisition framework to facilitate the development and reengineering of care plans in electronic format. In: Proc. of the Troms $\varnothing$ Telemedicine and eHealth Conference (TTeC-08). (June 2008)

5. Hernandez, C., Casas, A., Escarrabill, J., Alonso, J., Puig-Junoy, J., Farrero, E., Vilagut, G., Collvinent, B., Rodriguez-Roisin, R., Roca, J., partners of the CHRONIC project: Home hospitalisation of exacerbated chronic obstructive pulmonary disease patients. European Respiratory Journal 21 (2003) 58-67

6. Global initiative for chronic Obstructive Lung Disease: Global Strategy for the Diagnosis, Management and Prevention of Chronic Obstructive Pulmonary Disease. Executive Summary. http://www.goldcopd.com/ (2006)

7. The Task Force for the Diagnosis and Treatment of Chronic Heart Failure of the European Society of Cardiology: Guidelines for the diagnosis and treatment of chronic heart failure: executive summary (update 2005). European Heart Journal 26 (2005) 1115-1140

8. Peleg, M., Miksch, S., Seyfang, A., Bury, J., Ciccarese, P., Fox, J., Greenes, R., Hall, R., Johnson, P., Jones, N., Kumar, A., Quaglini, S., Shortliffe, E., Stefanelli, M.: Comparing computer-interpretable guideline models: A case-study approach. Journal of the American Medical Informatics Association 10 (2003) 52-68

9. de Clercq, P., Blom, J., Korsten, H., Hasman, A.: Approaches for creating computer-interpretable guidelines that facilitate decision support. Artificial Intelligence in Medicine 31 (2004) 1-27

10. Isern, D., Moreno, A.: Computer-based execution of clinical guidelines: a review. International Journal of Medical Informatics 77(12) (2008) 787-808

11. Sutton, D., Fox, J.: The syntax and semantics of the PROforma guideline modeling language. Journal of the American Medical Informatics Association 10(5) (2003) 433-443

12. Tallis Toolset. http://www.cossac.org/tallis/index.html. Date of access: May 2009 